\title{
Estimation of track dimensions obtained in Laser Metal Deposition-powder thanks to a semi-analytical model coupled to an Eulerian thermal simulation
}

\author{
Cécile Leroy-Dubief, Fabien Poulhaon and Pierre Joyot
}

\author{
Cécile Leroy-Dubief. Univ. Bordeaux, ESTIA INSTITUTE OF TECHNOLOGY, I2M, UMR 5295, F-64210 Bidart, France \\ Corresponding author: c.leroydubief@estia.fr \\ Fabien Poulhaon. Univ. Bordeaux, ESTIA INSTITUTE OF TECHNOLOGY, I2M, UMR 5295, F-64210 Bidart, France \\ Pierre Joyot. Univ. Bordeaux, ESTIA INSTITUTE OF TECHNOLOGY, I2M, UMR 5295, F-64210 Bidart, France
}

\begin{abstract}
Originally issued from cladding, the LMD-p process widens the field of possibilities in terms of manufacturing. Depending on the targeted application, the needs regarding the track geometry are different and the ability to adapt it is a key challenge. In LMD-p, the laser beam attenuation as well as the powder particles preheating are both determined by laser-powder interactions before the powder reaches the substrate. The track dimensions are directly correlated to the melt pool size: a larger pool will tend to capture more powder resulting in a higher deposition rate. The model presented here intends to determine, for a given working distance, the partition of energy, and to estimate the area of the generated melt pool and finally the dimensions of the deposited track. It is first based on a semi-analytical approach that models the powder distribution and calculates the transmitted power to both substrate and powder particles. The attenuated power density is then an input for a light Eulerian thermal simulation from which the contour of the molten zone is extracted. Several iterations are carried out to account for the energy loss caused by the heating and melting of the powder entering the pool. Lastly, the track dimensions are estimated from the stabilized melt pool configuration. Track geometries obtained with a BeAM® machine are compared to the model predictions. Such an approach opens very interesting perspectives in studying the influence of the working distance and its optimization for a given material and/or a given application.
\end{abstract}

Keywords. Additive Manufacturing, LMD-p Process, Thermal Eulerian Model, Semi-analytical Approach, Track Geometry

\section{Introduction}

Additive manufacturing is revolutionising the way metal parts are designed and manufactured. The Laser Metal Deposition - Powder (LMD-p) process enables applications such as cladding, part production and function addition. Each of these applications requires optimisation of machine parameters prior to manufacturing.

Originally, powder spraying technology was originally used only for cladding [1], [2], which explains why there is an abundance of literature on the subject [3]. Technological advances have made it possible to use a coaxial laser powder jet instead of a lateral one, which has led to the development of LMD-p technology itself, capable of manufacturing large volume parts [4]. The result is a more complex thermal history and phenomena due to the multiplication of layers. Depending on the machine manufacturer, the nozzles may have several diametrically opposed powder outlets (Trumpf $®$, $\mathrm{LENS}^{\mathrm{TM}}$ ) or a continuous $360^{\circ}$ ring $(\mathrm{BeAM} 囚)$ forming a powder cone in each case. The design of the head greatly influences the speed of the powder flow [4], in addition to the speeds of the various gases used (lens protection, oxidation shield and powder transport).

It soon became necessary to simulate the process in order to predict the geometry of the manufactured track on the one hand, and the thermal history of the material [5], on the other hand, from which its microstructure (metallurgical phases, grain size and morphology, porosities) [6], [7] and mechanical properties are derived, and finally to optimise the machine parameters [8]. Numerous empirical "ground rules" have emerged before a more formal approach in the form of analytical and/or numerical models was obtained [4]. The majority of analytical models are based on a 
decoupling of the heat flow brought by the laser beam and the powder flow (e.g. [9]). However, they do not take into account the fact that the powder that impacts the melt pool tends to cool it, which leads to an overestimation of the size of the melt pool [10]. This is why numerical models based on CFD ([11]-[13]), FEM ([14]-[16]) or on a combination of the 2 methods ([17]) have appeared to reproduce with a greater fidelity the different physical phenomena involved. In [18], Pinkerton confronts analytical model and numerical simulation in order to predict the dilution and the width of a bead. Both models are then subjected to experimental validation; the numerical method tends to overestimate the size of the bead while the analytical approach underestimates it. Perhaps the most accurate solution then lies in a combined numerical and analytical method such as Peyre [19], [20]. This is also the line of research of the work presented here.

To this end, a semi-analytical model was developed, representing the attenuation of the laser beam by powder particles. This provides the preheating temperature of the powder as well as the attenuated laser power impacting the substrate. These data serve then as an input to a thermal Eulerian simulation which aims to estimate the width and the height of the track. The results of this simulation are then compared to experimental data carried out on a BeAM® machine.

\section{Model description}

\subsection{General approach}

The purpose of the model presented here is to predict the width of a bead deposited by the LMD-p process. It is compared to track dimensions obtained on a $\mathrm{Be} A \mathrm{M} 囚$ machine, for different sets of parameters (laser power, deposition speed and powder flow rate) used. The general approach comprises three steps: the initial iteration consists of calculating the incident power, i.e. the effective laser power impacting the pool after powder attenuation, taking into account the losses induced by beam transport via a fibre. During this step, the temperature rise of the powder particles on their way to the melt pool is also estimated. The analytically calculated attenuated power is then used as an input to a steady-state thermal model, based on an Eulerian FEM formulation (Morfeo®). This second calculation step allows a first estimate of the size of the molten zone. This pool width cannot be considered equivalent to the width of the bead because the powder falling into the pool, which is colder, tends to shrink the melt area. This is why, using the semianalytical model, the powder distribution determines how much material enters the pool and at which temperature, so a sink term quantifies the energy used to melt these particles. A few iterations then lead to a stabilisation of the pool width, resulting from a compromise between keeping the pool molten and adding cold material. This dimension is then estimated as the width of the track ( $c f$. Fig. 1). 


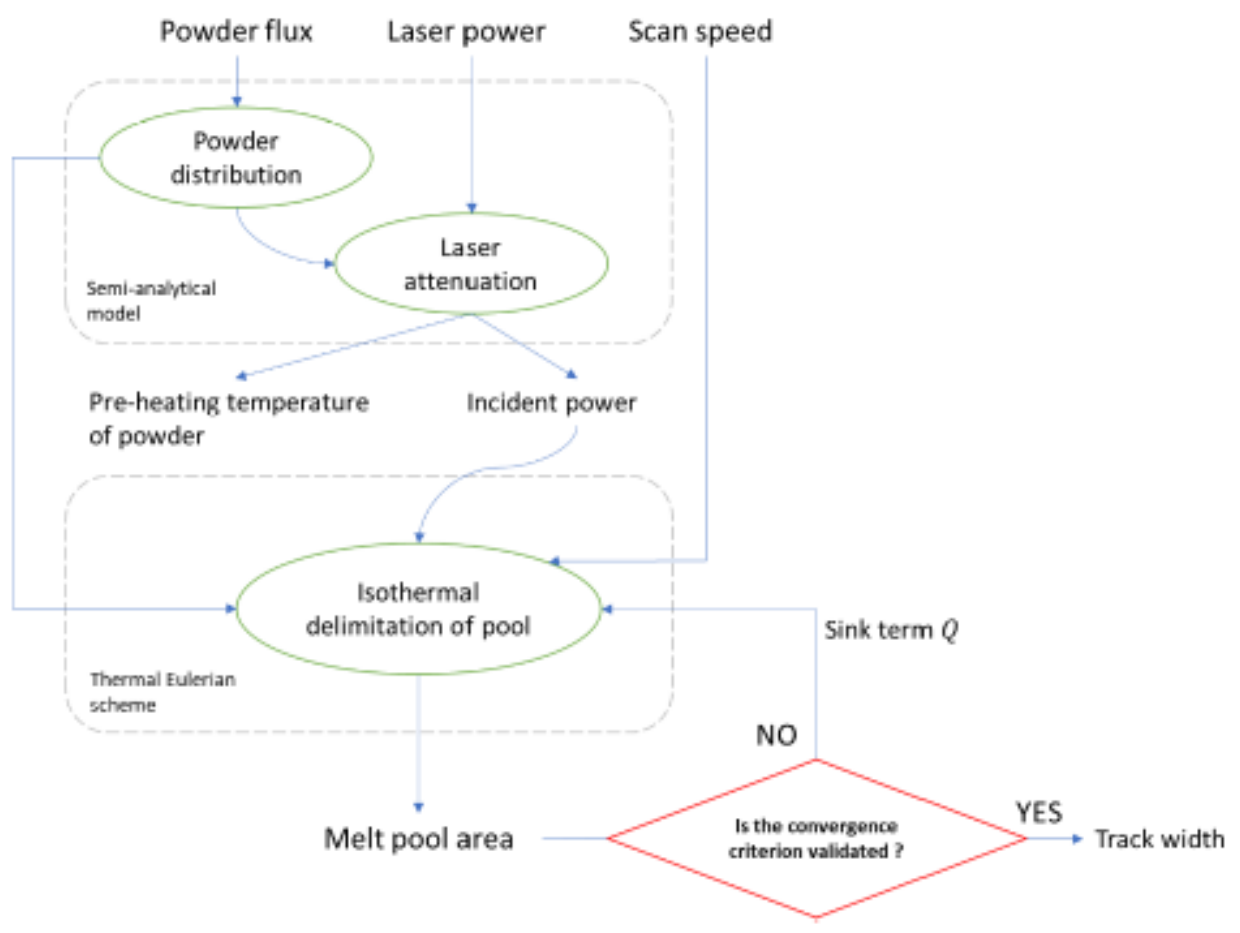

Fig. 1. Overview of the developed method combining a semi-analytical model and a thermal model

The BeAM has a $360^{\circ}$ continuous powder output. Its laser beam is defocused with its focal point above the substrate as shown in Fig. 2. A primary gas (argon) protects the optical lenses and is discharged through the same orifice as the laser; a second carrier gas allows the powder to be conveyed by giving it an initial velocity. The special feature of the machine is that a third gas is supplied to the first gas, which is used to shape the powder jet.

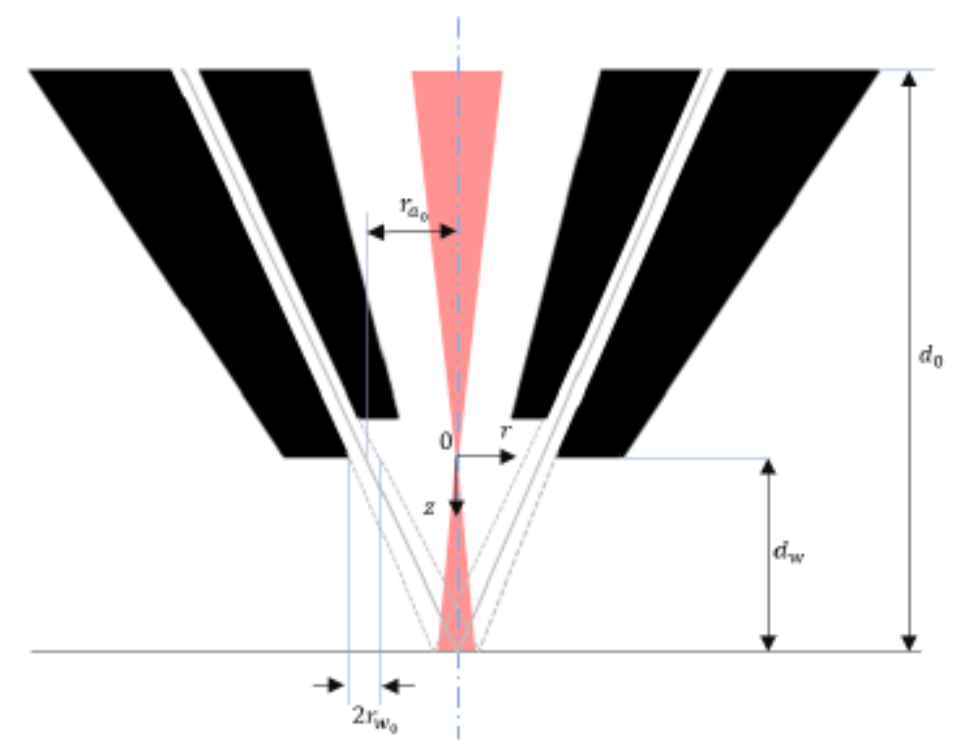


Estimation of track dimensions obtained in Laser Metal Deposition-powder thanks to a se...

Fig. 2. Schema of BeAM® nozzle

The assumptions used for the model developed are as follows:

- The flow is quasi-static in the semi-analytical model while the deposition speed is taken into account in an Eulerian thermal model. The particle velocity is assumed to be constant although this is not the case since the forming gas accelerates the particles. The effects of gravity and drag are neglected ([21], [22]).

- Powder particles are assumed to be spherical and of constant radius, as in Diniz Neto [23]; this tends to be true for powder produced by gas-atomisation [24].

- Interactions between particles such as collisions or shadow effects are neglected [25], power losses due to second reflections are also neglected [26].

- The laser beam is considered to be cylindrical with a radius of $L$ for the sake of simplicity, despite it is defocused (cf. Fig. 2); and its intensity distribution is considered to be top-hat (cf. Figure 4 in [24]). 
ESAFORM 2021. MS13 (Additive Manufacturing), 10.25518/esaform21.2488

Table 1. Nomenclature and values taken in the model 
Estimation of track dimensions obtained in Laser Metal Deposition-powder thanks to a se...

\begin{tabular}{|c|c|c|c|}
\hline Designation & Symbols & Values & Units \\
\hline Powder nozzle radius & $r_{a 0}$ & 1.45 & $m m$ \\
\hline Half powder nozzle outlet & $r_{w 0}$ & 0.26 & $m m$ \\
\hline Working distance & $d_{w}$ & 3.5 & $m m$ \\
\hline Height of powder flow & $d_{0}$ & 4.78 & $m m$ \\
\hline Powder mass flow rate & $F$ & & g.min $\min ^{-1}$ \\
\hline Powder distribution & $D_{r}$ & & $\mathrm{~kg} \cdot \mathrm{m}^{-2} \cdot \mathrm{s}^{-1}$ \\
\hline Laser power & $P_{0}$ & & $W$ \\
\hline Laser beam radius & $L$ & $3.18 \times 10^{-1}$ & $m m$ \\
\hline Initial laser intensity & $I_{0}$ & & $W \cdot m^{-2}$ \\
\hline Initial particle speed & $v$ & $1.76 \times 10^{2}$ & $m \cdot s^{-1}$ \\
\hline Component of $v$ in the $\mathrm{z}$ direction & $v_{Z}$ & & $m \cdot s^{-1}$ \\
\hline Powder particle radius & $r_{p}$ & 37.5 & $\mu m$ \\
\hline Density of particle material (at $298 \mathrm{~K}$ ) & $\rho$ & 4429 & $\mathrm{~kg} \cdot \mathrm{m}^{-3}$ \\
\hline Laser beam intensity & $I_{r}(z)$ & & $W . m^{-2}$ \\
\hline Incident laser power & $P_{i}$ & & $W$ \\
\hline Coordinates of particle from the nozzle outlet to $(Z, R)$ position & $\left(r^{\prime}, z^{\prime}\right)$ & & \\
\hline Position of powder particle & $(Z, R)$ & & \\
\hline Axial component of the velocity of a particle in the right path & $v_{Z}^{\prime}$ & & $m \cdot s^{-1}$ \\
\hline Absorptivity of powder particle & $\alpha$ & 0.4 & \\
\hline Specific heat capacity (at 298 K) & $c_{p}$ & 538 & $J \cdot \mathrm{kg}^{-1} \cdot K^{-1}$ \\
\hline Ambient temperature & $T_{0}$ & 298 & $K$ \\
\hline Temperature of particle at $(Z, R)$ position in the melt pool & $T_{\text {inPool }}$ & & $K$ \\
\hline Thermal conductivity (at $298 \mathrm{~K}$ ) & $k$ & 7.19 & $W \cdot m^{-1} K^{1}$ \\
\hline
\end{tabular}




\begin{tabular}{llll}
\hline Melting temperature & $T_{\text {fusion }}$ & 1898 & $K$ \\
\hline Specific latent heat of fusion & $L_{\text {fusion }}$ & $2.86 \times 10^{5}$ & $J . \mathrm{kg}^{-1}$ \\
\hline Sink term & $Q$ & $\mathrm{~kg} \cdot \mathrm{m}^{-2} \cdot \mathrm{s}^{-1}$ \\
\hline
\end{tabular}

\subsection{Semi-analytical schema}

Let's start by writing the radii $r_{a}$ and $r_{w}$ which define, respectively, the mean radius and the half-width of the powder jet ring at a distance $z$ :

$$
r_{a}(z)=\frac{d_{w}-z}{d_{w}} r_{a_{0}} \text { and } r_{w}(z)=\left(\frac{z}{d_{0}-d_{w}}+1\right) r_{w_{0}}
$$

As most papers suggest (e.g. [12], [13], [22]-[25]), the distribution of particles (5) within the powder jet can be represented by a $T E M_{01}{ }^{*}$ Gaussian function (cf. Figure 3.11 in [3]). To do so, two components $C_{1}(3)$ and $C_{2}(4)$, are calculated, both of which have units similar to an area:

$$
C_{1}(z)=\pi r_{w}^{2} e^{-2}\left(\frac{r_{a}}{r_{w}}\right)^{2} \text { and } C_{2}(z)=\pi r_{w} r_{a} \sqrt{2 \pi} \operatorname{erf}\left(\sqrt{2} \frac{r_{a}}{r_{w}}\right)
$$

The powder mass flow rate per unit area is then given by the following relationship and represented in Fig. 3:

$$
D_{r}(z)=\frac{F}{C_{1}+C_{2}}\left[e^{-2\left(\frac{r-r_{a}}{r_{w}}\right)^{2}}+e^{-2\left(\frac{r+r_{a}}{r_{w}}\right)^{2}}\right]
$$




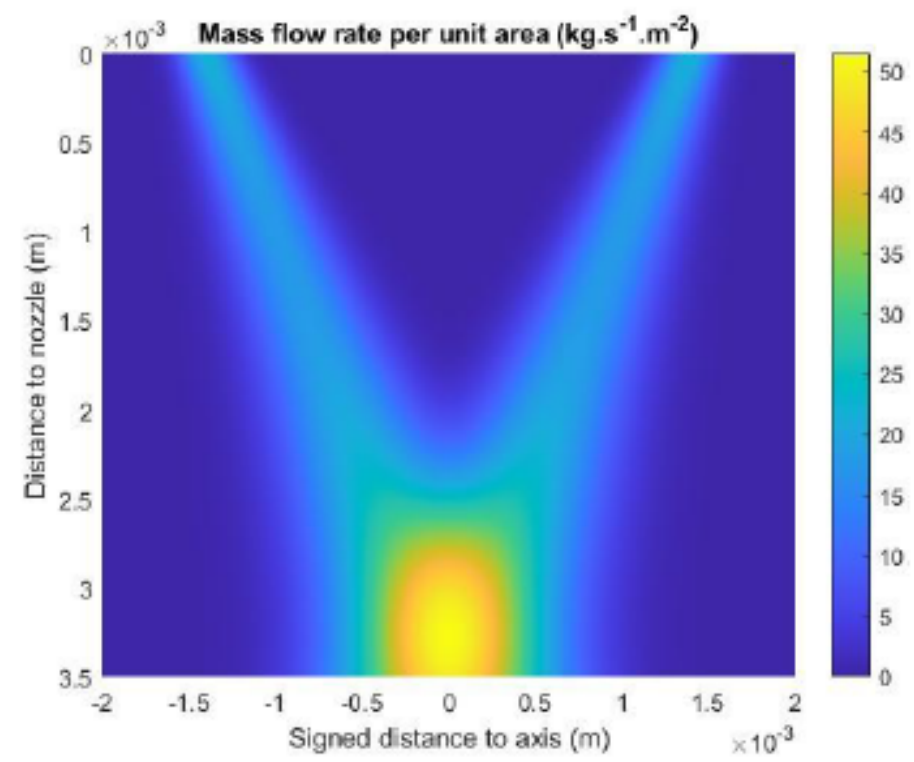

Fig. 3. Numerical representation of particles distribution within the powder flow

The laser intensity is considered to be uniform over the entire cross-section of the beam. Beer-Lambert's law expresses the intensity of the beam at a distance $\mathrm{z}$ from the nozzle exit [25]:

$$
I_{r}(z)=\frac{P_{0}}{\pi L^{2}} \exp \left(-\frac{3}{4 v_{z} r_{p} \rho} \int_{0}^{z} D_{r}(z) d z\right)
$$

With $v_{Z}$ being the velocity component in the $z$-direction of the powder particles.

By integrating the intensity $I_{r}\left(z=d_{w}\right)$ over the entire cross-section of the laser beam we obtain the incident power $P_{i}$ which impacts the melt pool:

$$
P_{i}=2 \pi \int_{0}^{L} r I_{r}\left(z=d_{w}\right) d r
$$

In order to predict the temperature to which the powder particles are heated before impacting the molten zone, it is necessary to express their trajectory using their coordinates $(Z, R)$. For the sake of simplicity, linear trajectories are considered here, although the different gases play a role in the deflection of the particles; however, as shown by Lin (Fig. 4 in [27]), the fact that the inner nozzle is slightly set inward from the outer nozzle tends to form a straight jet. The trajectory equations (8) can be reformulated as a function of $r$. 


$$
r^{\prime}(z)=\left(\frac{d_{0} r_{a_{0}}}{d_{w}}-R\right)\left(\frac{z-z}{z+d_{0}-d_{w}}\right)+R \text { and } z^{\prime}(r)=\frac{z\left(r_{a_{0}} d_{0}-r d_{w}\right)+(R-r)\left(d_{0}-d_{w}\right) d_{W}}{r_{a_{0}} d_{0}-R d_{w}}
$$

On the other hand, the z component of the particle velocities (10) can be written as follows:

$$
v_{z}{ }^{\prime}(r)=\frac{\left(Z+d_{0}-d_{w}\right) v}{\sqrt{\left(Z+d_{0}-d_{w}\right)^{2}+\left(\frac{d_{0} r_{a_{0}}}{d_{w}}-R\right)^{2}}}
$$

The temperature of the particles [23] that fall into the pool, i.e. those whose coordinates are $Z=d_{w}$ and $0<R<L$, is finally estimated using the relation:

$$
T_{\text {inPool }}(R)=T_{0}+\frac{3 \alpha}{4 \rho c_{p} r_{p}}\left[\frac{1}{2 v_{z}^{\prime}} \int_{z^{\prime}(L)}^{d_{w}} I\left(z, r^{\prime}(z)\right) d z+\frac{1}{2 v_{z}^{\prime \prime}} \int_{z^{\prime \prime}(L)}^{d_{w}} I\left(z, r^{\prime \prime}(z)\right) d z\right]
$$

where $v_{z}{ }^{\prime \prime}$ and $z$ " are respectively the velocity and the altitude of the particles originating from the opposite powder stream.

The time during which the particles pass through the laser beam is not sufficient to reach their melting temperature; this is why a portion of the incident power is allocated to melt this colder added mass. This leads to introduce in the Eulerian thermal model, a sink term reflecting this phenomenon. This corrective term takes the form of a flux applied in the powder's impact zone, and of opposite sign to the heat flux brought by the laser. It is obtained by the following relationship:

$$
Q=D_{r}\left(d_{w}\right) *\left(\int_{\text {Tpreheating }}^{T \text { fusion }} c_{p}(T) d T+L_{\text {fusion }}\right)
$$

Where $L_{\text {fusion }}$ is the specific latent heat of fusion.

\subsection{Steady state Eulerian thermal model}

The incident power, noted $P_{i}$ estimated using the semi-analytical model, allows the definition of an equivalent heat source in the Eulerian thermal model (Morfeo $®$ ). In this model, the heat source is fixed and a section of material, initially at room temperature, moves at the deposition speed (travel speed). A stationary solution is obtained at a lower calculation cost (cf. Fig. 4). In addition, a localised refinement of the mesh in the heating zone guarantees greater precision in the heat input. 

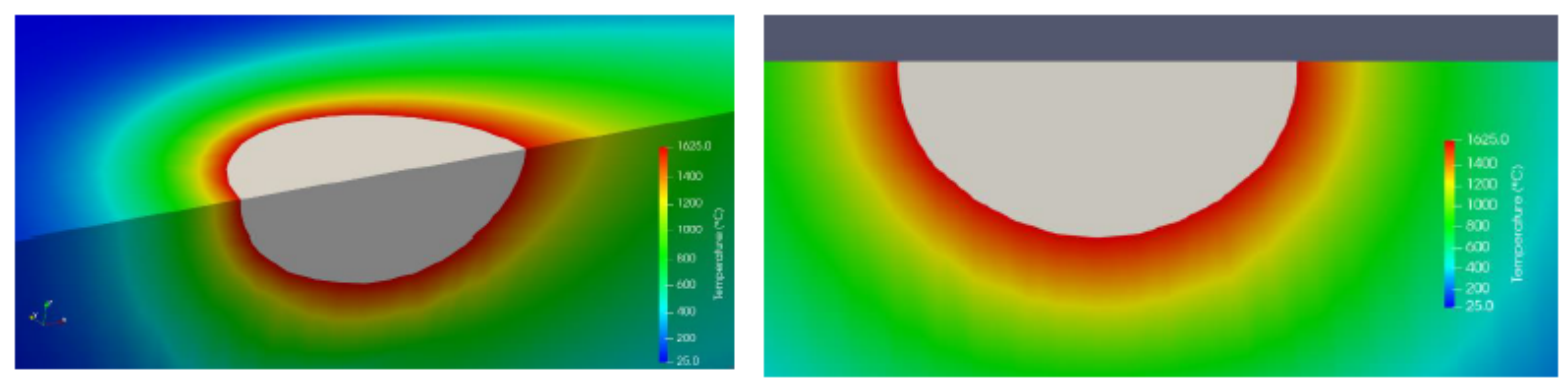

Fig. 4. Temperature field obtained from Eulerian model: melt pool is coloured in grey

This model takes into account the variation of the material properties $k, c p$ and $\rho$ with temperature. In addition, it considers heat loss by convection and radiation. A first calculation allows a rough estimate of the dimensions of the melted zone. The quantity of powder "captured" by the melt pool can then be estimated and the corrective term is calculated according to equation (12). The successive iterations then consist in adjusting the corrective term until the size of the pool predicted by the model converges (cf. Fig. 5).
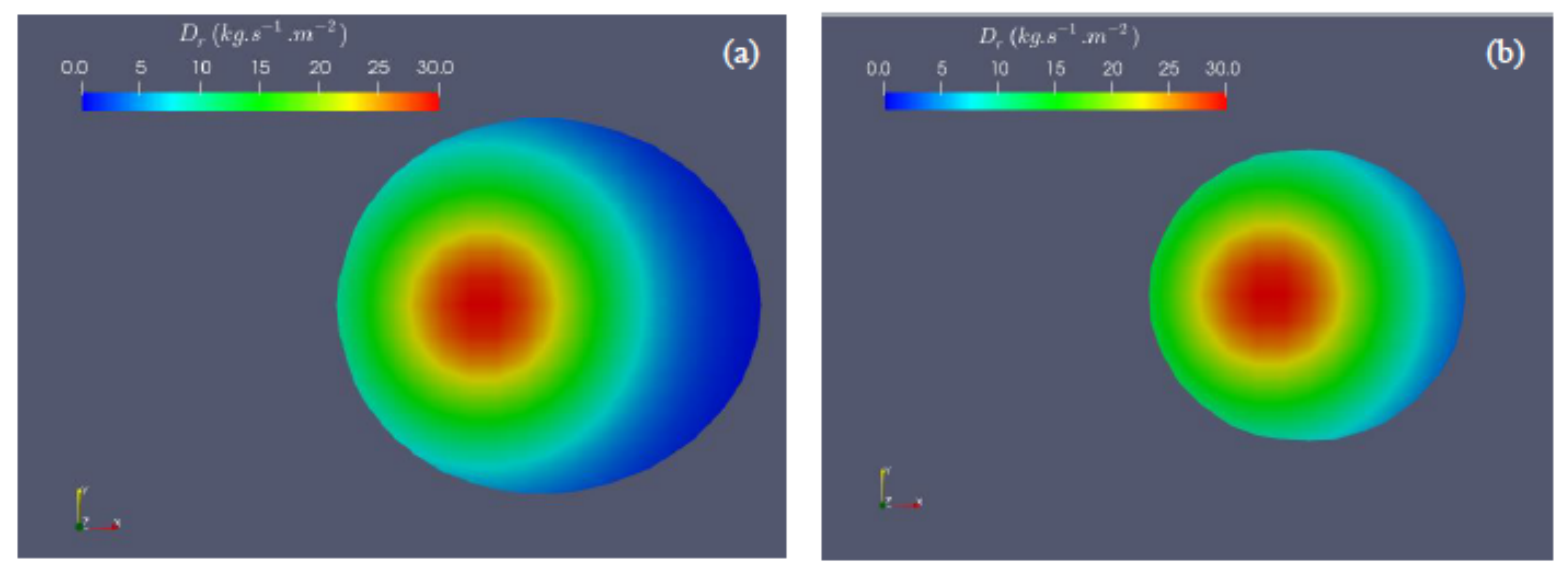

Fig. 5. Powder distribution spread over the surface of the pool (a) First iteration (initial), (b) Fifth iteration (final)

\section{Experimental data and validation}

The material adopted for this experiment is a Ti6Al4V powder (supplied by Tekna Inc.). The small nozzle configuration of the $\mathrm{BeAM} \otimes$ machine is used, the dimensions and values necessary for the simulation are provided in Table 1. 
Table 2. Set of machine parameters used

\begin{tabular}{|c|c|c|c|c|}
\hline Run number & Laser power $(\mathrm{W})$ & Incident power $(\mathrm{W})$ & Scan speed $\left(m s^{-1}\right)$ & Powder flow rate $\left(\mathrm{g} \mathrm{min}^{-1}\right)$ \\
\hline 1 & 660 & 570 & 1000 & 3.5 \\
\hline 2 & 360 & 236 & 1500 & 1.5 \\
\hline 3 & 660 & 570 & 2500 & 3.5 \\
\hline
\end{tabular}

For each set of parameters shown in Table 2, a total of 5 iterations were necessary to obtain a stabilised melt pool. Figure 6 illustrates the fluctuation of the predicted track width during the calculation.

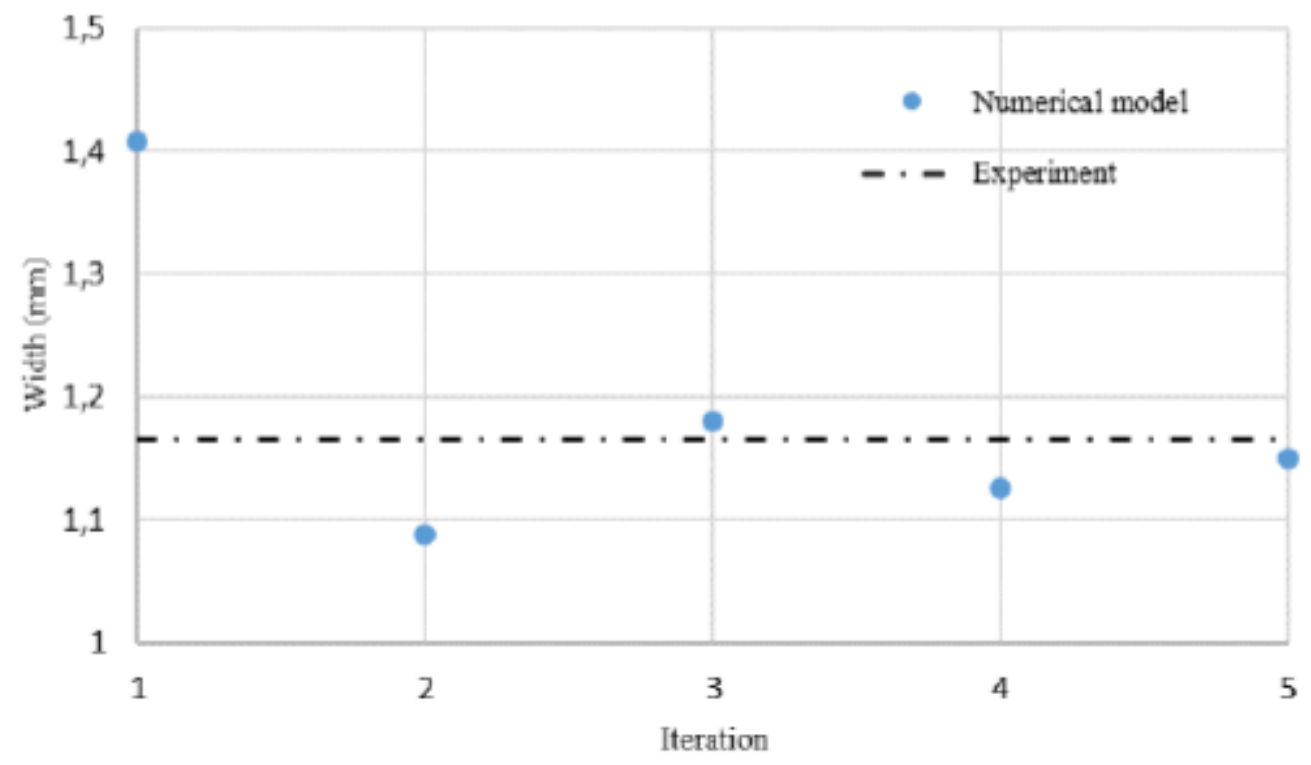

Fig. 6. Convergence of melt pool width (Run 1) after 5 iterations

After stabilisation of the track width, the cross-section area is estimated, considering the powder mass flow rate distribution and the travel speed. Both width and area are finally used to estimate the track height, by making the hypothesis that the cross-section exhibits a circular shape. Estimated width and height are presented in table 3.

Table 3. Experimental and simulated results 
Estimation of track dimensions obtained in Laser Metal Deposition-powder thanks to a se...

\begin{tabular}{llllll}
\hline \multirow{2}{*}{ Run number } & \multicolumn{3}{c}{ Track width $(\mu \mathrm{m})$} & \multicolumn{2}{c}{ Track height $(\mu \mathrm{m})$} \\
\cline { 2 - 6 } & Experiment & Model & Error $(\%)$ & Experiment & Model \\
\hline 1 & 1166 & 1150 & -1.37 & 577 & $575^{*}$ \\
\hline 2 & 740 & 712 & -3.78 & 179 & 177 \\
\hline 3 & 979 & 927 & -5.31 & 260 & 281 \\
\hline
\end{tabular}

* this height corresponds to the maximum value achievable with a semi-circle approximation, but it is underestimated.

The bead width resulting from the model is, in a general way, underestimated. The relative error increases when the melt pool size decreases. This can be explained by the fact that the mesh size is fixed: any partially melted element is considered as part of the pool, whether it is only slightly or completely melted. This feature can be improved with a remeshing strategy performed during the iterations. Moreover, in order to apply this model to wider melt pools (high energy densities), it seems necessary to include the dynamics of fluids within the melt pool (especially Marangoni effect) [20]. As for the height estimation, the circularity hypothesis provides relatively good trends. However, some phenomena like surface tensions and/or gravity effect tend to flatten the bead and to induce more specific cross-section forms, that cannot be captured with such a model.

\section{Discussion and conclusion}

A combined model based on a semi-analytical approach and a steady-state thermal Eulerian formulation has been developed to predict the height and width of a track deposited with LMD-p technology. Experimental validation has been carried out on a BeAM $\AA$ machine. Laser intensity attenuation as well powder preheating temperature are first estimated and used as input data for the calculation of the track dimensions. An iterative approach making use of a heat sink term has been successfully applied to calculate a stabilised melt-pool with a good approximation in a relatively short calculation time. However, a remeshing strategy capturing the actual contour of the melt pool at each iteration would be necessary to increase the accuracy of the track width approximation. Regarding the height, the prediction is less accurate due to gravity effects and surface tensions which are not considered in this work. Finally, in order to deal with larger energy densities, resulting in wide melt pools, the current model would require some adjustments, in order to account for the dynamics of the liquid material. One option as suggested by some authors, would be to consider an anisotropic thermal conductivity in the melt pool. Such an anisotropy could result from thermal gradient considerations, as it is the main cause for liquid material convection. This feature will be the object of future works.

\section{Acknowledgements}

This research project was undertaken with the assistance of resources and services from the ADDIMADOUR platform, Additive Manufacturing Solutions.

\section{Bibliography}

[1] J. Lin and W. M. Steen, 'An in-process method for the inverse estimation of the powder catchment efficiency during laser cladding', p. 8, 1998.

[2] J. Lin, 'A simple model of powder catchment in coaxial laser cladding', Optics \& Laser Technology, vol. 31, no. 3, pp. 233-238, Apr. 1999, doi: 10.1016/S0030-3992(99)00046-8.

[3] E. Toyserkani, A. Khajepour, and S. Corbin, Laser Cladding, CRC Press. Boca Raton: Taylor \& Francis, 2004. 
[4] A. J. Pinkerton, 'Laser direct metal deposition: theory and applications in manufacturing and maintenance', in Advances in Laser Materials Processing, Elsevier, 2010, pp. 461-491.

[5] M. Alimardani, E. Toyserkani, and J. P. Huissoon, 'Three-dimensional numerical approach for geometrical prediction of multilayer laser solid freeform fabrication process', Journal of Laser Applications, vol. 19, no. 1, pp. 14-25, Dec. 2006, doi: 10.2351/1.2402518.

[6] L. Costa, R. Vilar, T. Reti, and A. M. Deus, 'Rapid tooling by laser powder deposition: Process simulation using finite element analysis', Acta Materialia, vol. 53, no. 14, pp. 3987-3999, Aug. 2005, doi: 10.1016/j.actamat.2005.05.003.

[7] S. Liu and Y. C. Shin, 'Prediction of 3D microstructure and phase distributions of Ti6Al4V built by the directed energy deposition process via combined multi-physics models', Additive Manufacturing, vol. 34, p. 101234, Aug. 2020, doi: 10.1016/j.addma.2020.101234.

[8] A. Fathi, E. Toyserkani, A. Khajepour, and M. Durali, 'Prediction of melt pool depth and dilution in laser powder deposition', Journal of Physics. D, Applied Physics, vol. 39, no. 12, pp. 2613-2623, 2006, doi: 10.1088/0022-3727/ $39 / 12 / 022$.

[9] A. F. H. Kaplan and G. Groboth, Process Analysis of Laser Beam Cladding. .

[10] L. Han and J. Choi, 'Two Dimensional Modeling of Laser Cladding With Droplet Injection', Dec. 2008, pp. 183-188, doi: 10.1115/HT2003-47295.

[11] J. Choi, L. Han, and Y. Hua, 'Modeling and Experiments of Laser Cladding with Droplet Injection,"', Journal of Heat Transfer-Transactions of the ASME, pp. 978-986, 2005.

[12] H. Qi, J. Mazumder, and H. Ki, 'Numerical simulation of heat transfer and fluid flow in coaxial laser cladding process for direct metal deposition', Journal of Applied Physics, vol. 100, no. 2, p. 024903, Jul. 2006, doi: 10.1063/1.2209807.

[13] X. He and J. Mazumder, 'Transport phenomena during direct metal deposition', Journal of Applied Physics, vol. 101, no. 5, p. 053113, Mar. 2007, doi: 10.1063/1.2710780.

[14] M. Picasso and A. F. A. Hoadley, 'Finite element simulation of laser surface treatments including convection in the melt pool', Int Jnl of Num Meth for HFF, vol. 4, no. 1, pp. 61-83, Jan. 1994, doi: 10.1108/EUM0000000004031.

[15] P. Peyre, M. Dal, S. Pouzet, and O. Castelnau, 'Simplified numerical model for the laser metal deposition additive manufacturing process', Journal of Laser Applications, vol. 29, no. 2, p. 022304, May 2017, doi: 10.2351/1.4983251.

[16] X. Lu et al., 'Finite element analysis and experimental validation of the thermomechanical behavior in laser solid forming of Ti-6Al-4V', Additive Manufacturing, vol. 21, pp. 30-40, May 2018, doi: 10.1016/j.addma.2018.02.003.

[17] S. Marimuthu, R. M. Eghlio, A. J. Pinkerton, and L. Li, 'Coupled Computational Fluid Dynamic and Finite Element Multiphase Modeling of Laser Weld Bead Geometry Formation and Joint Strengths', Journal of Manufacturing Science and Engineering, vol. 135, no. 1, p. 011004, Feb. 2013, doi: 10.1115/1.4023240.

[18] A. J. Pinkerton, A. M. Kamara, K. Shah, S. Safdar, and L. Li, 'Three dimensional analytical and finite element methods for simulating a moving melt pool with mass addition', in Pacific International Conference on Applications of Lasers and Optics, Beijing, People’s Republic of China, 2008, pp. 369-374, doi: 10.2351/1.5057040.

[19] P. Peyre, P. Aubry, R. Fabbro, R. Neveu, and A. Longuet, 'Analytical and numerical modelling of the direct metal deposition laser process', J. Phys. D: Appl. Phys., vol. 41, no. 2, p. 025403, Jan. 2008, doi: 10.1088/0022-3727/41/ 
2/025403.

[20] J. I. Arrizubieta, A. Lamikiz, F. Klocke, S. Martínez, K. Arntz, and E. Ukar, 'Evaluation of the relevance of melt pool dynamics in Laser Material Deposition process modeling', International Journal of Heat and Mass Transfer, vol. 115, pp. 80-91, Dec. 2017, doi: 10.1016/j.ijheatmasstransfer.2017.07.011.

[21] A. J. Pinkerton and L. Li, 'An analytical model of energy distribution in laser direct metal deposition', Proceedings of the Institution of Mechanical Engineers, Part B: Journal of Engineering Manufacture, vol. 218, no. 4, pp. 363-374, Apr. 2004, doi: 10.1243/095440504323055498.

[22] Y.-L. Huang, G.-Y. Liang, J.-Y. Su, and J.-G. Li, 'Interaction between laser beam and powder stream in the process of laser cladding with powder feeding', Modelling Simul. Mater. Sci. Eng., vol. 13, no. 1, pp. 47-56, Jan. 2005, doi: 10.1088/ 0965-0393/13/1/004

[23] 0. 0. Diniz Neto and R. Vilar, 'Physical-computational model to describe the interaction between a laser beam and a powder jet in laser surface processing', Journal of Laser Applications, vol. 14, no. 1, pp. 46-51, Feb. 2002, doi: $10.2351 / 1.1436485$.

[24] A. J. Pinkerton, 'An analytical model of beam attenuation and powder heating during coaxial laser direct metal deposition', J. Phys. D: Appl. Phys., vol. 40, no. 23, pp. 7323-7334, Dec. 2007, doi: 10.1088/0022-3727/40/23/012.

[25] J. Liu, L. Li, Y. Zhang, and X. Xie, 'Attenuation of laser power of a focused Gaussian beam during interaction between a laser and powder in coaxial laser cladding', J. Phys. D: Appl. Phys., vol. 38, no. 10, pp. 1546-1550, May 2005, doi: $10.1088 / 0022-3727 / 38 / 10 / 008$.

[26] W. Devesse, D. De Baere, and P. Guillaume, 'Modeling of laser beam and powder flow interaction in laser cladding using ray-tracing', Journal of Laser Applications, vol. 27, no. S2, p. S29208, Feb. 2015, doi: 10.2351/1.4906394.

[27] J. Lin, 'Numerical simulation of the focused powder streams in coaxial laser cladding', Journal of Materials Processing Technology, vol. 105, no. 1-2, pp. 17-23, Sep. 2000, doi: 10.1016/S0924-0136(00)00584-7.

PDF automatically generated on 2021-05-20 06:27:39

Article url: https://popups.uliege.be/esaform21/index.php?id=2488

published by ULiège Library in Open Access under the terms and conditions of the CC-BY License (https://creativecommons.org/licenses/by/4.0) 